\title{
APPROXIMATE SOLUTIONS OF FREDHOLM-TYPE INTEGRAL EQUATIONS
}

\author{
A. T. LONSETH ${ }^{1}$
}

1. Introduction. The integral equation whose approximate solution will be discussed here is the linear nonhomogeneous equation of Fredholm type and second kind

$$
x(s)-\int_{0}^{1} K(s, t) x(t) d t=y(s), \quad 0 \leqq s \leqq 1,
$$

where the functions $K(s, t)$-the "kernel" -and $y(s)$ are known. The question posed is this: Given an approximate method for solving (1.1), how can one estimate the error?

Since late in the nineteenth century the importance of (1.1) in mathematical physics has been recognized, along with that of

$$
x(s)-\lambda \int_{0}^{1} K(s, t) x(t) d t=0, \quad 0 \leqq s \leqq 1,
$$

in which one is to determine values of $\lambda$ (proper values) such that a continuous solution $x(s) \not 0$ exists. Typical problems leading to equations like (1.1) are the Dirichlet and Neumann problems of potential theory; to (1.2), time-dependent problems in elastic vibration and heat flow, by "separating out" the time.

It is now fifty years since Fredholm published his distinguished paper in Acta Mathematica [10], ${ }^{2}$ in which he gave the first detailed account of the existence and multiplicity of solutions of (1.1) and (1.2). Few mathematical publications have stimulated so much further work. Several papers appeared in which physical problems were set up in terms of integral equations (elasticity, gas theory, etc.); some were on numerical solutions; most of the many theoretical papers followed the now familiar trend toward greater generality and abstraction. Hilbert recognized analogies with Euclidean geometry, except that now space was infinite-dimensional [13]; through Fréchet and F. Riesz this led to Banach spaces and more rarefied

An address delivered before the Missoula meeting of the Society on June 20, 1953, by invitation of the Committee to Select Hour Speakers for Far Western Sectional Meetings; received by the editors December 15, 1953.

1 This paper was prepared with financial assistance from the Office of Ordnance Research, U. S. Army, under terms of Contract DA-04-200-ORD-177 with Oregon State College.

${ }^{2}$ Numbers in brackets refer to the bibliography at the end of the paper. 
concepts. Such contemporary objects as "abstract spaces," "spectral theory," and "normed rings" are among the grandchildren of the Fredholm theory.

Despite their apparent remoteness from numerical work, some of these abstract ideas lie very close to our question of error-limitation. This is true of such simple metric notions as the norm (or length) of a function and the bound of an integral transformation $K$ defined by

$$
K x=\int_{0}^{1} K(s, t) x(t) d t .
$$

It is convenient to write (1.1) in the abbreviated form

$$
(I-K) x \equiv x-K x=y
$$

and to speak of $x$ and $y$ as "vectors" in some "linear space," such as the totality of functions which are continuous on $0 \leqq s \leqq 1$, or of those whose squares are integrable thereon. More details may be found in $[2],[15]$, or [19].

It will be assumed throughout that (1.1) has a unique solution $x(s)$ for given $y(s)$. Thus we may speak of the inverse $(I-K)^{-1}$ of operation $I-K$. All functions and numbers will be assumed real, but everything here extends to the complex case with only trivial alterations.

2. Three classes of methods. The three categories for which error bounds will be derived do not cover all ways of attacking (1.1), nor are they mutually exclusive. However, most of the frequently used procedures will be included.

Class I. The kernel $K(s, t)$ is replaced by an approximation of such form that the resulting equation can be solved exactly. Usually the new kernel is one of "finite rank" such as

$$
(\alpha) \sum_{i=1}^{n} \phi_{i}(s) \psi_{i}(t) \quad \text { or } \quad(\beta) \sum_{i=1}^{n} \sum_{j=1}^{n} a_{i j} \phi_{i}(s) \psi_{j}(t),
$$

where the $\phi$ 's and $\psi$ 's are known functions, preferably tractable; with such a kernel (1.1) reduces to an ordinary system of linear equations $[11 ; 26 ; 3]$. For example, with $K(s, t)$ replaced by $(\alpha)$ the integral equation becomes

$$
x_{n}(s)-\sum_{i=1}^{n} \phi_{i}(s) \int_{0}^{1} \psi_{i}(t) x_{n}(t) d t=y(s) .
$$

We multiply in turn by $\psi_{1}(s), \cdots, \psi_{n}(s)$ and integrate over $(0,1)$, getting $n$ linear equations for the numbers 


$$
p_{i}^{(n)}=\int_{0}^{1} \psi_{i}(t) x_{n}(t) d t, \quad i=1, \cdots, n
$$

If this system is nonsingular, its solution substituted into (2.1) gives exactly

$$
x_{n}(s)=y(s)+\sum_{i=1}^{n} p_{*}^{(n)} \phi_{i}(s) .
$$

The problem then is to get some kind of limitation for the difference $x(s)-x_{n}(s)$. Various such bounds have been published by Tricomi [27], Akbergenov [1], Kantorovich [15; 16] and Lonseth [19]. These error-limitations do not require that the approximating kernel be of finite rank, but merely that it be sufficiently close to $K(s, t)$ in some sense.

Class II. The equation is unchanged; instead, the "best" near-solution of form

$$
x_{n}(s)=\phi_{0}(s)+\sum_{i=1}^{n} a_{i} \phi_{i}(s)
$$

is determined, where the $\phi$ 's are known functions. (Boundary conditions imposed by $K(s, t)$ and $y(s)$ may be absorbed in $\phi_{0}(s)$, while the other $\phi$ 's satisfy the corresponding "homogeneous" conditions.) Everything now depends on the meaning of "best," and with different criteria we have different methods. One criterion for choice of the $a$ 's is to minimize the integral

$$
Q\left(a_{1}, \cdots, a_{n}\right)=\int_{0}^{1}\left\{x_{n}(s)-\int_{0}^{1} K(s, t) x_{n}(t) d t-y(s)\right\}^{2} d s .
$$

This "method of least squares" leads to a system of linear equations for $a_{1}, \cdots, a_{n}$ and is quite effective. Another method is based on the principle of moments (Galerkin's method): the $a$ 's are chosen so that

$$
x_{n}(s)-\int_{0}^{1} K(s, t) x_{n}(t) d t-y(s)
$$

is orthogonal to $\phi_{1}(s), \cdots, \phi_{n}(s)$ on $(0,1)$. That is, the $a$ 's satisfy $n$ linear equations

$$
\int_{0}^{1} \phi_{i}(s)\left\{x_{n}(s)-\int_{0}^{1} K(s, t) x_{n}(t) d t-y(s)\right\} d s=0, \quad i=1, \cdots, n .
$$

If $K(s, t) \equiv K(t, s)$, the method of moments coincides with a minimum principle of Rayleigh and Ritz. Various generalizations have been 
considered. We refer those who are interested to the books by Kravchuk [17] and by Kantorovich and V. I. Krylov [16], and to journal articles by N. M. Krylov [18], Picone [25], and Oberg [22].

Class III. Iterative methods. Some iterative methods are schemes for solving (1.1) as it stands, with given $y(s)$; others yield the inverse of the operator $I-K$ which in (1.1) transforms the unknown $x$ into the known $y$. C. Neumann's expansion is the prototype of methods of both sorts. Formally it says that, whatever $y$, the solution is given by

$$
x=y+K y+K^{2} y+\cdots,
$$

where $K^{n} y$ is the result of operating $n$ times on $y$ with $K$; this is the same as saying that the inverse

$$
(I-K)^{-1}=I+K+K^{2}+\cdots .
$$

Thus $x$ is approximated by $x_{n}$ which is recursively defined by

$$
\begin{aligned}
x_{0} & =y, \\
x_{n+1} & =y+K x_{n} \quad \text { if } n=0,1, \cdots .
\end{aligned}
$$

Likewise, $(I-K)^{-1}$ is approximated by $S_{n}$ where

$$
\begin{aligned}
S_{0} & =I, \\
S_{n+1} & =I+K S_{n} \quad \text { if } n=0,1, \cdots .
\end{aligned}
$$

Faster schemes for getting $(I-K)^{-1}$ will also be described in $\$ 6$.

Perhaps the most important method not explicitly discussed here is that in which the integral

$$
\int_{0}^{1} K(s, t) x(t) d t
$$

is replaced by an approximation (trapezoidal, Simpson's, Gauss', etc.) and $x(t)$ is approximately determined only at finitely many points. This idea was Fredholm's heuristic guide in [10]. It has been used by Nyström in [21] and elsewhere, and the error has been investigated by Ostrowski [24] and Kantorovich [15; 16]. Gradient methods (such as "steepest descent") may also prove useful, particularly for symmetric kernels.

Actual application of these methods may require numerical integrations, or solution of a system of linear equations, or both. In the illustrations at the end of this paper the necessary quadratures could be carried out exactly, and the linear systems were of such low orders that a desk-computer was quite adequate. Such favorable examples 
cannot always be expected, however, and a modern, high-speed automatic calculator may be desirable, or even necessary. Solving a highorder system of linear equations can be quite a problem. It is discussed comprehensively in G. E. Forsythe's paper [29].

3. Metric notions. The length or norm $\|x\|$ of a function $x(s)$, $0 \leqq s \leqq 1$, may be defined in various ways. A natural adaptation of the ordinary Euclidean length of an $n$-dimensional vector is

$$
\|x\|=\left(\int_{0}^{1} x^{2}(s) d s\right)^{1 / 2}
$$

With this quadratic norm, a linear combination of two functions with finite norm also has finite norm; furthermore,

$$
\begin{aligned}
\|x\| & \geqq 0 ; \\
\|a x\| & =|a| \cdot\|x\| \quad \text { if } a \text { is a constant; } \\
\|x+y\| & \leqq\|x\|+\|y\| .
\end{aligned}
$$

These properties characterize norms generally: they hold if one defines, for $x(s)$ continuous on $0 \leqq s \leqq 1$,

$$
\|x\|=\max _{0 \leqq s \leqq 1}|x(s)|
$$

or, for $x(s)$ such that $|x(s)|^{p}$ is integrable,

$$
\|x\|=\left(\int_{0}^{1}|x(s)|^{p} d s\right)^{1 / p}, \quad p \geqq 1 .
$$

Error-estimates will be found in terms of norms. It is important to know what a linear transformation does to the norm of a function. Suppose that the functions $x(s)$ under consideration constitute a (real) normed linear space (i.e., if $x_{1}$ and $x_{2}$ are in the space, so is $a_{1} x_{1}$ $+a_{2} x_{2}$, where $a_{1}$ and $a_{2}$ are any real numbers; for every $x,\|x\|$ exists). Suppose further that $T$ is a linear transformation of the type known as linear operators (i.e., additive, homogeneous, such that $\|x\|$ finite. implies $\|T x\|$ finite, continuous in the sense that $\left\|x_{n}-x\right\| \rightarrow 0$ implies $\left.\left\|T x_{n}-T x\right\| \rightarrow 0\right)$. Then there exist $[2$, p. 54] non-negative numbers $M(T)$ and $m(T)$,

$$
M(T)=\underset{\|x\| \neq 0}{1 \mathrm{u} . \mathrm{b} .} \frac{\|T x\|}{\|x\|}, \quad m(T)=\underset{\|x\| \neq 0}{\mathrm{~g} .1 . \mathrm{b} .} \frac{\|T x\|}{\|x\|},
$$

which we call respectively the upper and the lower bounds of $T$. Evidently, whatever $x$, 


$$
m(T)\|x\| \leqq\|T x\| \leqq M(T)\|x\| .
$$

If the sum $T_{1}+T_{2}$ and the product $T_{1} T_{2}$ of two linear operators $T_{1}$ and $T_{2}$ are operators defined so that, for every $x,\left(T_{1}+T_{2}\right) x=T_{1} x$ $+T_{2} x$ and $\left(T_{1} T_{2}\right) x=T_{1}\left(T_{2} x\right)$, they are also bounded and

$$
M\left(T_{1}+T_{2}\right) \leqq M\left(T_{1}\right)+M\left(T_{2}\right), \quad M\left(T_{1} T_{2}\right) \leqq M\left(T_{1}\right) M\left(T_{2}\right) .
$$

If $T$ has the bounded inverse $T^{-1}$, then [19]

$$
M\left(T^{-1}\right) m(T)=1 .
$$

If $T=I-K$ and $M(K)<1$,

$$
1 /\{1+M(K)\} \leqq M\left\{(I-K)^{-1}\right\} \leqq 1 /\{1-M(K)\},
$$

inequalities which we shall use later.

The exact calculation of $M(K)$ is usually out of the question. If $K(s, t)$ is symmetric and the norm is the quadratic (3.1), $M(K)$ is the reciprocal of the smallest $\lambda$ solving the characteristic problem (1.2). With the same norm and a nonsymmetric kernel, $M(K)$ is $\mu^{-1 / 2}$, where $\mu$ is the smallest number such that there is a $w(s) \not \equiv 0$ satisfying

$$
w(s)-\mu \int_{0}^{1}\left\{\int_{0}^{1} K(r, t) K(r, s) d r\right\} w(t) d t=0 .
$$

These may not be easy to find. An upper bound to $M(K)$ comes from the Schwarz inequality:

$$
M(K) \leqq\left(\int_{0}^{1} \int_{0}^{1} K^{2}(s, t) d s d t\right)^{1 / 2} .
$$

With $\|x\|=\max |x(s)|$,

$$
M(K) \leqq \max _{0 \leqq s \leqq 1} \int_{0}^{1}|K(s, t)| d t .
$$

With $\|x\|=\int_{0}^{1}|x(s)| d s$,

$$
M(K) \leqq \int_{0}^{1} \max _{0 \leqq t \leqq 1}|K(s, t)| d s .
$$

With the Minkowski norm (3.3) for $p>1$, Hölder's inequality yields

$$
M(K) \leqq\left\{\int_{0}^{1}\left[\int_{0}^{1}|K(s, t)|^{q} d t\right]^{p / q} d s\right\}^{1 / p}
$$

where $p^{-1}+q^{-1}=1$.

4. Error-bound for kernel-replacement. Suppose that $K(s, t)$ is 
replaced by $K(s, t)+\kappa(s, t)$, and write $x(s)+\xi(s)$ for the solution of the new equation. Then, briefly, we have

$$
x+\xi-(K+\kappa)(x+\xi)=y
$$

while

$$
x-K x=y
$$

so

$$
\xi-K \xi=\kappa x+\kappa \xi .
$$

If now $(I-K)^{-1}=I+R$,

$$
\xi=(I+R)(\kappa x+\kappa \xi) .
$$

If $M\{(I+R) \kappa\}<1$, we can limit $\|\xi\|$; for

$$
\|\xi\| \leqq M\{(I+R) \kappa\}(\|x\|+\|\xi\|),
$$

and consequently

$$
\|\xi\| \leqq M\left\{(I+R)_{\kappa}\right\}\|x\| /\left[1-M\left\{(I+R)_{\kappa}\right\}\right] .
$$

Since

$$
M\{(I+R) \kappa\} \leqq M(I+R) M(\kappa),
$$

the foregoing will be valid for sufficiently small $M(\kappa)$. And if $M(\kappa)$ $<1-M(K)$, we find that

$$
\|\xi\| \leqq M(\kappa)\|x\| /\{1-M(K)-M(\kappa)\} .
$$

We can also write down a point-wise bound for $|\xi(s)|$, no matter which of the norms in $\S 3$ is used. For instance, with the Euclidean norm (3.1) we see by applying the Schwarz inequality to (4.1) that

$$
\begin{aligned}
|\xi(s)| \leqq & \left(\int_{0}^{1} K^{2}(s, t) d t\right)^{1 / 2}\|\xi\| \\
& +\left(\int_{0}^{1} \kappa^{2}(s, t) d t\right)^{1 / 2}(\|x\|+\|\xi\|) .
\end{aligned}
$$

Into (4.4) we must substitute the bound (4.2) for $\|\xi\|$ and use

$$
\|x\| \leqq M(I+R)\|y\|,
$$

in which $M(I+R) \leqq 1 /\{1-M(K)\}$ if $M(K)<1$.

A quite different bound, based on Fredholm's solution-formula and the Hadamard determinant inequality, was published in 1924 by Tricomi [27]. Akbergenov [1] in 1935 got essentially the results of this section for the norms (3.1) and (3.2), the latter of which is 
given by Kantorovich and Krylov [16, p. 157]. Related but complicated expressions are to be found in [15] as well. The present discussion is based on [19].

5. Error-bound for methods of the second class. Here we are looking for the "best" near-solution to (1.1) that lies in a certain finitedimensional functional manifold. Let

$$
x_{n}(s)=\phi_{0}(s)+\sum_{i=1}^{n} a_{i} \phi_{i}(s)
$$

be an arbitrary function in this manifold; let the selected values of $a_{1}, \cdots, a_{n}$-determined by least squares, moments, or any other method-be $\tilde{a}_{1}, \cdots, \tilde{a}_{n}$. The corresponding "solution" $\tilde{x}_{n}$ does not necessarily satisfy (1.1) but rather

$$
\tilde{x}_{n}-K \tilde{x}_{n}-y=\tilde{r}_{n},
$$

in which the residual $\tilde{r}_{n}(s)$ can be calculated a posteriori. Subtracting from (5.1) the true equation satisfied by the still unknown $x(s)$, we get

$$
\tilde{x}_{n}-x-K\left(\tilde{x}_{n}-x\right)=\tilde{r}_{n}
$$

so that with $(I-K)^{-1}=I+R$ as before,

$$
\tilde{x}_{n}-x=(I+R) \tilde{r}_{n}
$$

and for an appropriate norm

$$
m(I+R)\left\|\tilde{r}_{n}\right\| \leqq\left\|\tilde{x}_{n}-x\right\| \leqq M(I+R)\left\|\tilde{r}_{n}\right\| .
$$

If $M(K)<1,(5.3)$ becomes, because of (3.8),

$$
\left\|\tilde{r}_{n}\right\| /\{1+M(K)\} \leqq\left\|\tilde{x}_{n}-x\right\| \leqq\left\|\tilde{r}_{n}\right\| /\{1-M(K)\} .
$$

Thus in this case we not only get the desired upper bound for the error-norm, but also a positive lower bound, if $\left\|\tilde{r}_{n}\right\|>0$. (The lower bound holds even if $M(K) \geqq 1$.) That there should be such a lower bound may at first seem surprising, but actually it is to be expected: we are trying to pick out of a subspace a vector which cannot be expected to be there, and whose "distance" from each vector of the subspace (usually) exceeds some positive quantity. The same considerations apply more generally to solution of $T x=y$, where $T$ is linear (possibly a differential or integro-differential operator): if $T$ is itself bounded, $m\left(T^{-1}\right)=1 / M(T)$ and $\left\|\tilde{x}_{n}-x\right\|$ is bounded away from zero; if $T$ has a bounded inverse, we get an upper bound for $\left\|\tilde{x}_{n}-x\right\|$.

The local error can be limited much as in $\$ 4$ (see 4.4). Or it may be 
better to observe from (5.2) that

$$
x(s)-\tilde{x}_{n}(s)+\tilde{r}_{n}(s)=-\int_{0}^{1} K(s, t)\left[\tilde{x}_{n}(t)-x(t)\right] d t .
$$

Now if, e.g., the quadratic norm is being used, we have by the Schwarz inequality

$$
\left|x(s)-\tilde{x}_{n}(s)+\tilde{r}_{n}(s)\right| \leqq k(s)\left\|\tilde{x}_{n}-x\right\|, \quad 0 \leqq s \leqq 1,
$$

where

$$
k(s)=\left(\int_{0}^{1}[K(s, t)]^{2} d t\right)^{1 / 2}
$$

and (5.3) can be used to limit $\left\|\tilde{x}_{n}-x\right\|$. Thus we have $x(s)$ lying between the two functions

$$
\tilde{x}_{n}(s)-\tilde{r}_{n}(s) \pm k(s)\left\|\tilde{x}_{n}-x\right\|, \quad 0 \leqq s \leqq 1 .
$$

If the quadratic norm is used, the method of least squares is seen to have unique advantages. One is that the criterion for goodness is precisely that $\left\|\tilde{r}_{n}\right\|$ be the minimum; accordingly inequalities (5.3) would be most favorable. This does not prove, of course, that least squares is actually more accurate than any other similar method. Probably no such categorical statement is possible. Nevertheless, a method for which one can claim a certain accuracy has an advantage over methods for which one must claim less.

Another advantage which "least squares" has over other methods in this class consists in the relative ease of computing $\left\|\tilde{r}_{n}\right\|$-still the quadratic norm. In principle, $\tilde{r}_{n}(s)$ can always be computed from (5.1) after $x_{n}$ has been determined; it must then be squared and integrated, which may involve some rather nasty computation. But for least squares there is an alternative method based on "Bessel's identity":

$$
\left\|\tilde{r}_{n}\right\|^{2}=\left\|y-\phi_{0}\right\|^{2}-\sum_{i=1}^{n} \tilde{a}_{i}\left(y-\phi_{0}, T \phi_{i}\right),
$$

in which the inner products

$$
\left(y-\phi_{0}, T \phi_{i}\right)=\int_{0}^{1}\left(y-\phi_{0}\right)\left(\phi_{i}-K \phi_{i}\right) d s, \quad i=1, \cdots, n,
$$

are the constant right members of the equations determining $\tilde{a}_{1}, \cdots, \tilde{a}_{n}$. Thus the only new computation is that of $\left\|y-\phi_{0}\right\|^{2}$.

It should be pointed out that the simple considerations of this section are actually quite general; the $\tilde{x}_{n}$ in (5.1) can be any kind of 
approximation to the true $x$, and (5.3) holds provided that the symbols have meaning.

6. Error-bounds for iterative methods. If one writes (1.1) as

$$
\begin{aligned}
x & =y+K x \\
& =y+K(y+K x)=y+K y+K^{2} x \\
& =y+K y+K^{2} y+K^{3} x, \text { etc. }
\end{aligned}
$$

it is not hard to guess that if $M(K)<1$, so that $\left\|K^{n} x\right\| \rightarrow 0$, the sequence of vectors $\left\{x_{n}\right\}$, defined by

$$
x_{n}=y+K y+\cdots+K^{n-1} y,
$$

will converge to the solution $x$ of (1.1), in the sense that $\left\|x-x_{n}\right\| \rightarrow 0$. In fact,

$$
\left\|x-x_{n}\right\| \leqq M^{n}(K)\|y\| /\{1-M(K)\} .
$$

Also, if $S_{n}=I+K+\cdots+K^{n-1}$,

$$
M\left\{(I-K)^{-1}-S_{n}\right\} \leqq M^{n}(K) /\{1-M(K)\} .
$$

Instead of such a purely iterative approach, one might get an initial approximate solution by least squares or some other procedure and then wish to improve it by iteration. Suppose $\tilde{x}(s)$ is such an approximation, and write

$$
\tilde{x}-K \tilde{x}=y+\tilde{r} .
$$

The point-wise bounds in (5.7) suggest that $\tilde{x}(s)-\tilde{r}(s)$ may be a fair approximation to the true $x(s)$; we define $\tilde{x}_{1}(s)=\tilde{x}(s)-\tilde{r}(s)$, so that

$$
\tilde{x}_{1}-\tilde{x}=-\tilde{r},
$$

then define a sequence $\left\{\tilde{x}_{n}\right\}$ of further approximations by

$$
\tilde{x}_{k+1}-\tilde{x}_{k}=K^{k}(-\tilde{r}), \quad k=1, \cdots,
$$

the idea being to reduce $\|\tilde{r}\|$, already small, by operating with $K$, $M(K)<1$. Adding all such differences through $k=n-1$, we see that

$$
\tilde{x}_{n}-\tilde{x}=\sum_{k=0}^{n-1} K^{k}(-\tilde{r}), \quad \quad K^{0}=I .
$$

On the other hand, by subtracting (1.1) from (6.3) we observe that $\tilde{x}-x$ satisfies equation

$$
\tilde{x}-x-K(\tilde{x}-x)=\tilde{r},
$$

whence 


$$
\tilde{x}-x=\sum_{k=0}^{\infty} K^{k} \tilde{r} .
$$

Addition of (6.4) and (6.5) leads to the inequality

$$
\left\|\tilde{x}_{n}-x\right\| \leqq M^{n}(K)\|\tilde{r}\| /\{1-M(K)\} .
$$

For approximating the inverse $(I-K)^{-1}$, a more rapidly convergent iterative scheme than Neumann's can be based on an identity which Euler [8, p. 335] used in a problem on partitions, namely

$$
1 /(1-x)=(1+x)\left(1+x^{2}\right)\left(1+x^{4}\right) \cdots\left(1+x^{2 k}\right) \cdots,
$$

which holds if $|x|<1$. The $n$th partial product

$$
\prod_{k=0}^{n-1}\left(1+x^{2 k}\right)=\sum_{k=0}^{2 n-1} x^{k}
$$

so that for $I-K$, with

$$
H_{n}=(I+K)\left(I+K^{2}\right) \cdots\left(I+K^{2 n-1}\right),
$$

we have

$$
(I-K)^{-1}-H_{n}=K^{2 n}+K^{2 n+1}+\cdots, \quad M(K)<1,
$$

and $[20]$

$$
M\left\{(I-K)^{-1}-H_{n}\right\} \leqq M^{2 n}(K) /\{1-M(K)\} .
$$

The product expression (6.7) for $H_{n}$ can be replaced by a recursion formula. Clearly $H_{n+1}=H_{n}\left(I+K^{2 n+1}\right)=H_{n}\left\{2 I-\left(I-K^{2 n+1}\right)\right\}$, or

$$
H_{n+1}=H_{n}\left\{2 I-(I-K) H_{n}\right\},
$$

the final expression coming immediately from (6.7) on pre-multiplying each member by $I-K$. This technique was suggested by Ostrowski [23] for equations of Volterra type, and has been used for matrices by several authors.

A hierarchy of still more rapidly convergent iterative schemes can be constructed along the lines of Euler's identity. ${ }^{3}$ Next in line would be the identity

$$
\begin{aligned}
1 /(1-x) & =\left(1+x+x^{2}\right)\left(1+x^{3}+x^{6}\right)\left(1+x^{9}+x^{18}\right) \cdots \\
& =\prod_{k=0}^{\infty}\left(1+x^{36}+{ }^{2 \cdot 3 k}\right),
\end{aligned}
$$

${ }^{3}$ Added later. I am grateful to R. D. James and Leo Moser for helpful suggestions. 
valid if $|x|<1$. Generally, we have

$$
1 /(1-x)=\prod_{k=0}^{\infty}\left(\sum_{j=0}^{m-1} x^{j m^{k}}\right) .
$$

With $m=3$ and operators instead of numbers, we write

$$
H_{n}^{(3)}=\prod_{k=0}^{n-1}\left(I+K^{3 k}+K^{2 \cdot 3 k}\right) .
$$

Then one can show that

$$
(I-K)^{-1}-H_{n}^{(3)}=K^{3^{n}}+K^{3^{n}+1}+\cdots ;
$$

so

$$
M\left\{(I-K)^{-1}-H_{n}^{(3)}\right\} \leqq M^{3^{n}}(K) /\{1-M(K)\} .
$$

A recursion formula like (6.9) is also obtainable, but it is not so simple. From

$$
H_{n+1}^{(3)}=H_{n}^{(3)}\left(I+K^{3^{n}}+K^{2 \cdot 3^{n}}\right)
$$

and

$$
(I-K) H_{n}^{(3)}=I-K^{3^{n}}
$$

(the latter identity follows immediately from (6.13)), we get

$$
\begin{aligned}
H_{n+1}^{(3)} & =H_{n}^{(3)}\left\{I+\left[I-(I-K) H_{n}^{(3)}\right]+\left[I-(I-K) H_{n}^{(3)}\right]^{2}\right\} \\
& =H_{n}^{(3)}\left\{3 I-3(I-K) H_{n}^{(3)}+\left[(I-K) H_{n}^{(3)}\right]^{2}\right\},
\end{aligned}
$$

which is considerably more complicated than (6.9) or the simple relation

$$
H_{n+1}^{(1)}=I+K H_{n}^{(1)} \quad\left(H_{n}^{(1)}=S_{n}\right)
$$

which characterizes Neumann's expansion. The higher-order methods suggested by (6.11) with $m>3$ would be increasingly cumbersome.

Bodewig $[4 ; 5]$ has also discussed such rapidly convergent processes. It is interesting to observe that in [5] he refers to Euler's extension $[9$, p. 422] of Newton's method.

A different modification of the Neumann scheme was proposed by Wiarda and extended by $\mathrm{H}$. Bückner and $\mathrm{C}$. Wagner; details may be found in Bückner's monograph [6]-which contains much on the general subject of solving integral equations, particularly (1.2)-and Wagner's paper [28]. 
7. Numerical illustrations. ${ }^{4}$ Some of the methods described above have been tried out on the equation

$$
x(s)+\int_{0}^{1} K(s, t) x(t) d t=s^{2}, \quad 0 \leqq s \leqq 1,
$$

where

$$
K(s, t)= \begin{cases}s(1-t) & \text { if } 0 \leqq s \leqq t \leqq 1 \\ t(1-s) & \text { if } 0 \leqq t \leqq s \leqq 1 .\end{cases}
$$

This integral equation corresponds to the simple boundary value problem

$$
x^{\prime \prime}(s)-x(s)=2, \quad x(0)=0, \quad x(1)=1 .
$$

However, the exact solution will not be used in checking the approximate solutions for accuracy.

Kernel (7.1) has characteristic values $n^{2} \pi^{2}(n=1,2, \cdots)$; so with the quadratic norm $M(K)=1 / \pi^{2}$; but even without this information it follows from the Schwarz inequality that $M(K) \leqq 1 /(90)^{1 / 2}<1 / 9$. Hence

$$
9 / 10<m(I+K)<M(I+K)<9 / 8 .
$$

To $n^{2} \pi^{2}$ corresponds $\sin n \pi s$ as characteristic function; hence the spectral representation of $K(s, t)$ is its Fourier sine series

$$
K(s, t)=2 \sum_{n=1}^{\infty} \frac{\sin n \pi s \sin n \pi t}{n^{2} \pi^{2}} .
$$

Four solution-procedures will be summarized: (a) $K(s, t)$ is replaced by one, two, or three terms of (7.3); (b) least squares; (c) Galerkin's method of moments; (d) least squares followed by iteration. It should be remembered that all the upper bounds are pessimistic.

Example (a). With $K(s, t)$ replaced by just the first term from its series representation (7.3), the solution

$$
x_{1}(s)=s-0.034832 \sin \pi s
$$

is found. From inequality (4.3), with $M(K)$ computed from Bessel's identity, it is found that with the quadratic norm

$$
\left\|x-x_{1}\right\| \leqq 0.017 \text {. }
$$

The point-wise bound, from (4.4), satisfies in $0 \leqq s \leqq 1$

\footnotetext{
${ }^{4}$ The computations in this section were carried out by Mr. Thomas L. Glahn.
} 


$$
\begin{aligned}
\left|x(s)-x_{1}(s)\right| & \leqq 0.0098\left(s-s^{2}\right)+0.520\left(\frac{s^{2}(1-s)^{2}}{3}-\frac{2}{\pi^{4}} \sin ^{2} \pi s\right)^{1 / 2} \\
& \leqq 0.027 .
\end{aligned}
$$

Similar computations for $n=2$ and $n=3$ were carried out. The normerrors are bounded as follows:

$$
\left\|x-x_{2}\right\| \leqq 0.00822, \quad\left\|x-x_{3}\right\| \leqq 0.00501 .
$$

Kantorovich [16, pp. 160-161] applies Akbergenov's error bound with norm $\max |x(s)|$ to the equation

$$
x(s)-\int_{0}^{1 / 2}(\sin s t) x(t) d t=1+s^{-1}\left(\cos \frac{s}{2}-1\right),
$$

and with kernel sin $s t$ replaced by $s t-s^{3} t^{3} / 6$ shows that the pointwise error does not exceed $10^{-6}$. This excellent result is possible mainly because the kernel is analytic, with alternating power series in $s t$, and because the interval is rather short. It is not obvious how his inequality could be applied to the kernel (7.1). Kernels which are Green's functions for differential operators are not analytic, but frequently their squares are integrable, so the quadratic norm has a chance of being useful.

Example (b). "Least squares" was applied with $\phi_{0}(s) \equiv 0, \phi_{k}(s)$ $=s^{k-1}$ if $k \geqq 1$. With $n=2$, it was found that

$$
x_{2}(s)=-0.186785+0.983500 s ;
$$

with (5.4)

$$
0.0764<\left\|x-x_{2}\right\|<0.0955 .
$$

With $n=3$, it was found that

$$
x_{3}(s)=0.006903-0.144901 s+1.128397 s^{2},
$$

and now

$$
0.00280 \leqq\left\|x-x_{3}\right\| \leqq 0.00349 .
$$

It is clear that the norm of the error is 0.003 to three place accuracy.

EXAMPLE (c). The moment method with $n=3$ gave

$$
x_{3}(s)=0.006853-0.144819 s+1.128423 s^{2},
$$

with

$$
0.00286 \leqq\left\|x-x_{3}\right\| \leqq 0.00358 .
$$

EXAmple (d). A single iteration of the second sort described in $\S 6$ 
was applied to the $x_{2}(s)$ found in (b) above. This led to the approximation

$$
\tilde{x}_{2}(s)=-0.070524 s+0.906607 s^{2}+0.163917 s^{3},
$$

with upper bound (6.6)

$$
\left\|x-\tilde{x}_{2}\right\|<0.0106 \text {. }
$$

Added in proof (August 23, 1954). Since this paper was written, three related error limitations have been found on the Oregon State College project. The first, by T. L. Glahn, is for a recursive scheme which determines successive coefficients in Fredholm's two series for the resolvent kernel. The second, by L. B. Rall, applies to the iterative processes of G. Wiarda, H. Bückner, C. Wagner, and P. A. Samuelson. The third, by G. T. Thompson, gives a close bound for Bateman's method as applied to boundary problems for self-adjoint ordinary linear differential equations of second order.

\section{BIBLIOGRAPHY}

1. I. A. Akbergenov, On the approximate solution of Fredholm's equation and determination of its characteristic values (Russian), Mat. Sbornik vol. 42 (1935) pp. 679697; German summary, p. 698.

2. S. Banach, Théorie des opérations linéaires, Warsaw, 1932.

3. H. Bateman, On the numerical solution of linear integral equations, Proc. Roy. Soc. London Ser. A vol. 100 (1922) pp. 441-449.

4. E. Bodewig, Bericht über die verschiedenen Methoden zur Lösung eines Systems linearer Gleichungen mit reellen Koeffizienten (IV), Neder. Akad. Wetensch. vol. 51 (1948) pp. 53-64.

5. - Konvergenztypen und das Verhalten von Approximationen in der Nähe einer mehrfachen Wurzel einer Gleichung, Zeitschrift für Angewandte Mathematik und Mechanik vol. 29 (1949) pp. 44-51.

6. H. Bückner, Die praktische Behandlung von Integral-Gleichungen, Ergebnisse der angewandte Mathematik vol. 1, Berlin-Göttingen-Heidelberg, 1952.

7. P. D. Crout and F. B. Hildebrand, $A$ least square procedure for solving integral equations by polynomial approximation, Journal of Mathematics and Physics vol. 20 (1941) pp. 310-335.

8. L. Euler, Introductio in analysin infinitorum, vol. 1, Opera Omnia, Ser. 1, vol. 8, Leipzig-Berlin, 1922.

9. - Institutiones calculi differentialis, Opera Omnia, Ser. 1, vol. 10, Leipzig-Berlin, 1913.

10. I. Fredholm, Sur une classe d'équations fonctionnelles, Acta Math. vol. 27 (1903) pp. 365-390.

11. E. Goursat, Sur un cas élementaire de l'équation de Fredholm, Bull. Soc. Math. France vol. 35 (1907) pp. 163-173.

12. E. Hellinger and $\mathrm{O}$. Toeplitz, Integralgleichungen und Gleichungen mit unendlich vielen Unbekannten, Encyklopädie math. Wissenschaften II C 13; U.S. reprint 1953, Chelsea. 
13. D. Hilbert, Grundzïge einer allgemeinen Theorie der linearen Integralgleichungen, Leipzig-Berlin, 1912; U. S. reprint 1953, Chelsea.

14. F. B. Hildebrand, Methods of applied mathematics, New York, 1952.

15. L. V. Kantorovich, Functional analysis and applied mathematics (Russian), Uspehi Matem. Nauk vol. 3 (1948) pp. 89-185; English translation by C. D. Benster, ed. by G. E. Forsythe, National Bureau of Standards, Los Angeles, 1952.

16. L. V. Kantorovich and V. I. Krylov, Approximate methods of higher analysis (Russian), 3d ed., Moscow-Leningrad, 1950. [Chapter II of this excellent book is entitled Approximate solution of integral equations of Fredholm. An English translation of the book has been made by C. D. Benster, under G. E. Forsythe's editorial direction, and is available at the Institute for Numerical Analysis, University of California, Los Angeles.]

17. M. F. Kravchuk (M. Krawtshouk), Application of the method of moments to solution of linear differential and integral equations (Ukrainian), Kiev, 1932.

18. N. M. Krylov, Sur différents procédés d'intégration approchée en physique mathématique, Ann. Fac. Sci. Univ. Toulouse vol. 27 (1925) pp. 153-186; vol. 29 (1927) pp. 167-199.

19. A. T. Lonseth, The propagation of error in linear problems, Trans. Amer. Math. Soc. vol. 62 (1947) pp. 193-212.

20. - An extension of an algorithm of Hotelling, Proceedings of the (first) Berkeley Symposium on Mathematical Statistics and Probability, Berkeley-Los Angeles, 1949, pp. 353-358.

21. E. J. Nyström, Über die praktische Auflösung von Integralgleichungen mit Anwendungen auf Randwertaufgaben, Acta Math. vol. 54 (1930) pp. 185-204.

22. E. N. Oberg, The approximate solution of integral equations, Bull. Amer. Math. Soc. vol. 41 (1935) pp. 276-284.

23. A. Ostrowski, Sur une transformation de la série de Liouville-Neumann, C. R. Acad. Sci. Paris vol. 203 (1936) p. 602.

24. - Sur l'approximation du dêterminant de Fredholm par les déterminants des systèmes d'équations linéaires, Arkiv för Matematik, Astronomi och Fysik vol. 26A (1938) pp. 1-15.

25. M. Picone, Sul metodo delle minime potenze ponderate e sul metodo di Ritz per il calcolo approssimato nei problemi della fisica-matematica, Rend. Circ. Mat. Palermo vol. 52 (1928) pp. 225-253.

26. E. Schmidt, Zur Theorie der linearen und nicht-linearen Integralgleichungen (II), Math. Ann. vol. 64 (1907) pp. 161-174.

27. F. Tricomi, Sulla risoluzione numerica delle equazioni integrali di Fredholm, Atti della Accademia Nazionale dei Lincei Rendiconti. Classe di Scienze Fisiche, Matematiche e Naturali (5) vol. 33 (1924) $1^{\circ}$ sem., pp. 483-486, $2^{\circ}$ sem., pp. 26-30.

28. C. Wagner, On the solution of Fredholm integral equations of second kind by iteration, Journal of Mathematics and Physics vol. 30 (1951) pp. 23-30.

The following paper on solving linear systems of algebraic equations will be helpful in connection with some of the methods discussed here. It contains an extensive bibliography.

29. G. E. Forsythe, Solving linear equations can be interesting, Bull. Amer. Math. Soc. vol. 59 (1953) pp. 299-329.

The Oregon State College 